\title{
Aproximación al origen de la COPE: la radio parroquial en Sevilla
}

institucionales.us.es/ambitos/

\section{Pedro Domínguez Fernández}

English Version: Approach to the origin of COPE: the parish radio in Seville

La irrupción de las emisoras parroquiales en Sevilla a mediados del siglo XX tuvo su importancia en la historia de Sevilla al ser las únicas que ofrecían noticias independientes, informaban con libertad política y sindical y permitían abrir nuevos horizontes, trayendo contenidos de Ámerica, al margen de difundir la importancia laica en el mundo. Este fue el origen del actual Grupo COPE, una sociedad anónima cuyo objetivo sigue siendo apostar por la difusión cultural, informativa, religiosa y social, y

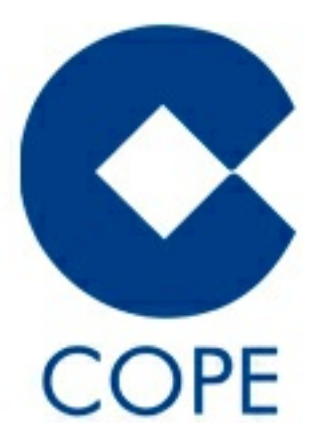
referente en el periodismo radifónico español. El presente escrito analiza el estado actual de este gigante mediático.

Palabras clave: radio parroquial, cadena COPE, emisora laica, medios de comunicación, Sevilla.

Abstract: The irruption of the parish stations in Seville in the middle of the twentieth century had its importance in the history of Seville being the only ones that offered independent news, informed with political and union freedom and allowed to open new horizons bringing contents of Ámerica, to the margin of diffusing the secular importance in the world. This was the origin of the current COPE Group, a limited company whose objective continues being a cultural promoter as well as the diffusion of informational, religious and social contents, and a reference in Spanish radio journalism. This writing analyzes the current state of this media giant.

Keywords: parish radio, COPE, secular radio station, media, Seville.

\section{BREVE INTRODUCCIÓN: LA RADIO EN SEVILLA. LAS PRIMERAS EMISIONES}

"En Sevilla, la radio lleva casi un siglo pegada a su día a día". La certera afirmación es de Antonio Yélamo, director de la Cadena SER Andalucía y de Radio Sevilla, y simplifica a la perfección el papel preeminente que la radio ha jugado en el contexto social e informativo de la ciudad durante gran parte del siglo XX y la proyección con la que se introdujo en el XXI.

Sevilla tiene un especial protagonismo en los inicios de la radio en España. En el verano de 1924 nacía en la capital andaluza EAJ-5, Radio Club Sevillano, mientras que en 1925 comenzaba a emitir la segunda emisora de la ciudad, EAJ-17, Radio Sevilla. Ambas 
emisoras se unen en 1927 naciendo Unión Radio Sevilla, ya desde entonces, y hasta hoy, en la calle Rafael González Abreu.

A lo largo de estos años, los momentos en los que la radio ha sido fundamental en la historia y en el día a día de la ciudad son innumerables. Siempre se mencionan las soflamas de Queipo de Llano a través de Radio Sevilla, aunque también habría que recordar que en 1932 emitió el bando militar del general golpista José Sanjurjo contra el gobierno de la Segunda República.

Fueron años "dorados" para la radio en la ciudad, convertida en casi el único medio de comunicación de masas en ausencia de la televisión. Hasta principios de los años 50 , Radio Sevilla era la única emisora en la ciudad. No fue hasta 1951 cuando Radio Nacional de España ${ }^{[1]}$ tuvo emisora en la ciudad y cuando aparecían las radios sindicales y las primeras emisoras parroquiales, que dieron origen al objeto de estudio y análisis de este trabajo: la Cadena COPE.

\section{EL GERMEN DE COPE: LAS EMISORAS PARROQUIALES. DE RADIO VIDA A RADIO POPULAR}

La existencia de radios parroquiales en España tuvo más importancia de la que en un principio pueda dársele, por pensar que su función era puramente pastoral y doctrinal. Su creación supuso un gran cambio sobre todo a partir del Concilio Vaticano II (iniciado en 1959), porque al ser emisoras de la Iglesia eran las únicas que podían dar noticias independientes del Parte de Radio Nacional: entre las noticias puramente eclesiales, van a empezar a propagar, promovidas por el Concilio, la libertad política, sindical, la importancia de los laicos en el mundo, o incluso las nuevas músicas procedentes de América que se colaban a través de las bases militares.

En Sevilla, en 1955, el Padre Manuel Linares, de la Compañía de Jesús, inició junto a un grupo de jóvenes - “los chicos de la congregación"-la apasionante tarea de poner en marcha una nueva emisora de radio en Sevilla vinculada a la Iglesia. Radio Vida nació el 26 de febrero de 1955 y sus emisiones, durante los primeros meses, se limitaban a 30 minutos diarios, pasando luego a una hora, cuatro y seis diarias, subiendo también la potencia de unos 50 vatios a los posteriores dos kilovatios cuando lograron profesionalizar sus instalaciones.

Radio Vida fue especialmente impulsada por la Congregación Mariana Universitaria de la Inmaculada y San Luis Gonzaga ("Los Luises"). No es casual que fuera el carisma de sus fundadores, la propia idiosincrasia jesuita y la juventud de sus primeros componentes los que hicieran de esta nueva radio un soplo de modernidad en aquella Sevilla de mitad de los años cincuenta del pasado siglo XX, frente a Radio Sevilla — tan vinculada en la memoria colectiva a Queipo de Llano y sus soflamas propagandísticas - y a Radio Nacional de España —unida al régimen-.

Erigida contra toda norma oficial imperante en la época, por un decreto fechado el 20 de mayo de 1955 y firmado por el arzobispo coadjutor, José María Bueno Monreal, el primer centro de emisiones fue la calle Trajano, en las instalaciones del número 39. El 25 de octubre de 1959, el ya Cardenal firmó los estatutos de fundación de la "Asociación Piadosa Radio Vida". Posteriormente, tras un breve paso por la huerta del Colegio Portaceli, 
trasladaría su centro emisor y sus nuevos estudios al número 24 de la calle Vírgenes. Es la "mítica" localización en la que generaciones de sevillanos sitúan aún una emisora que tuvo como principal objetivo apostar por la difusión cultural, informativa, religiosa y social de la ciudad, con programas como Vida de Espectáculos, Ámbito Olímpico, Ellas, Es grande ser joven, Noche del Sábado o Verano Club, por citar tan solo algunos ejemplos de programas que marcaron un antes y un después en la "encorsetada" programación radiofónica de la época. Especial mención merece Saeta, el programa en emisión de más antigüedad ${ }^{22]}$ en la radiodifusión española, cuyo espíritu sigue vivo más de medio siglo después de su primera emisión. Con la inminencia de la Expo'92, Radio Popular trasladó sus instalaciones a las nuevas —y desde entonces su última sede — instalaciones de la calle Rioja.

Respecto a su desarrollo como cadena nacional, dos hechos importantes se producen el 28 de noviembre de 1959, cuando se creó el Plan Nacional de Radiodifusión de la Iglesia; y a comienzos de los años 1960, cuando la Comisión Episcopal de Medios de Comunicación Social, presidida por el Arzobispo de Pamplona, Enrique Delgado Gómez, crea el Secretariado de Comunicación Social con el fin de reorganizar y legalizar —la inmensa mayoría de radios episcopales carecían de licencia- las emisoras de la Iglesia que, por aquel entonces, eran más de doscientas en toda España. La Comisión encarga al dominico Javier Sacristán García aplicar el Plan Transitorio de Ondas Medias, puesto en marcha por el Ministerio de Información y Turismo [3], a las emisoras episcopales.

Dentro de la negociación entre el Ministerio y la Cadena de Ondas Episcopales se le asigna a la Iglesia cincuenta emisoras en Onda Media (una por provincia) con el compromiso de emitir simultáneamente en Frecuencia Modulada. Sacristán ejerce ya como primer director general de COPE, cuyos estatutos fueron aprobados el 3 de diciembre de 1965, y en los años posteriores al acuerdo con el Ministerio se van instalando las nuevas emisoras y cerrando todas las parroquiales. Sin embargo, hay que destacar que solo permanece Radio Vida en Sevilla, que en adelante se llamará Radio Popular de Sevilla. Bajo la dirección de Sacristán, se empiezan a emitir programas en cadena y a gestionar la correspondiente publicidad. Posteriormente, el 9 de junio de 1971, se constituye la sociedad anónima COPE bajo la dirección general de José Luis Gago del Val.

El 9 de noviembre de 1978, COPE deja de ser una cadena institucional de la Iglesia y se convierte en sociedad civil, asumiendo el Estado la cuarta parte de sus acciones, al igual que de la SER. Esta situación se mantuvo hasta abril de 1992, cuando el gobierno vende el $5 \%$ de acciones que tenía aún de COPE y decidió no presentarse a posteriores ampliaciones de capital. En ese año había 81 emisoras en España y se encontraban a punto de adjudicarse 20 más.

Es la época de consolidación de la emisora, de la configuración de sus principales programas y de la vinculación a COPE de los más reconocidos profesionales de la radio nacional. Luis del Olmo, que abandonó Radio Nacional para incorporarse a COPE, Encarna Sánchez o Fermín Bocos fueron los referentes de la cadena durante décadas.

A principios de los años 90, la operación de PRISA para comprar las emisoras de su principal competidor en radio, Antena 3, y proceder a su cierre ${ }^{[4]}$ provocó el desembarco en COPE de los principales periodistas de esa cadena, con José María García y Antonio 
Herrero a la cabeza, lo que supuso, tras la marcha de Luis del Olmo [5], un importante empuje para COPE. La muerte de Encarna Sánchez (1996) y Antonio Herrero (1998), y la marcha de José María García a Onda Cero supusieron para la emisora un nuevo freno en términos de audiencia y repercusión social.

Durante los primeros años del siglo XXI, COPE marcó su parrilla con polémicas y provisionalidad. El continuo ir y venir de profesionales como Luis Herrero o José Apezarena (apenas estuvieron un par de temporadas, por diferentes motivos, al frente de sus respectivos espacios en la emisora) y los continuos enfrentamientos dialécticos con el gobierno socialista de Rodríguez Zapatero o con la Generalitat de Cataluña de locutores como Federico Jiménez Losantos o César Vidal supusieron una importante "crisis" de identidad —hubo quienes reprochaban a una emisora de ideario católico el tono de sus profesionales-y de audiencia.

Conflictos como el protagonizado con el Estudio General de Medios, a raíz de la infiltración de periodistas dirigidos por José Antonio Abellán para demostrar la presuntas irregularidades que se cometían en la medición de audiencias, provocó la expulsión de COPE del EGM (suspendida cautelarmente por los tribunales) y un enfrentamiento judicial que finalmente se resolvió con un acuerdo entre COPE y el EGM, asumiendo la emisora el pago de 125.000 euros en compensación por los gastos judiciales generados.

En 2009, la emisora prescinde de Losantos, Vidal y Herrero (que inician el proyecto profesional de esRadio) e inicia una transición hacia un nuevo modelo de programación, configuración del equipo de profesionales, enfoque y difusión editorial, adaptando el espíritu de COPE a los nuevos tiempos. El paso de periodistas como Ernesto Sáenz de Buruaga, Ignacio Villa o Ramón García no logra consolidar los respectivos espacios de la parrilla. Para intentar mejorar la audiencia, que había sufrido un bajón importante, la Cadena COPE contrata a una consultora sueca, Radio Intelligence, que diseña el modelo de emisora que actualmente conocemos en cuanto a distribución de espacios en la parrilla. Se aumentan la duración de los programas, se eliminan o reducen franjas locales, se apuesta por rejuvenecer la audiencia y se apuesta firmemente por los espacios deportivos con la llegada a COPE, desde la SER, de todo el equipo formado por Paco González, Manolo Lama y Pepe Domingo Castaño. Los cambios propuestos por Radio Intelligence se tradujeron en un aumento de la audiencia de 2012 a 2014, acercándose a los dos millones de oyentes según el EGM en ese periodo.

En 2015, la Cadena realiza su nueva y última gran renovación de espacios en la emisora. La llegada de Carlos Herrera desde Onda Cero, para dirigir cada mañana Herrera en COPE, la apuesta de Ángel Expósito, que deja la dirección de Mediodía COPE, al frente de La Tarde, la consolidación de Juan Pablo Colmenarejo en la franja nocturna con La Linterna, y, posteriormente, el fichaje de Juanma Castaño para dirigir El Partidazo de COPE, han inducido un crecimiento constante de la audiencia de la emisora episcopal. Según datos del último EGM en julio de 2017, 6.227.000 oyentes escuchan a diario las emisoras del Grupo COPE.

En la temporada 2016/2017, la Cadena COPE acrecentó su apuesta por una emisión local independiente a través de la creación de COPE+. La intención de Radio Intelligence era mantener las emisoras conectadas en cadena el mayor tiempo posible, por lo que se dio 
opción a las emisoras locales a desdoblar a través de otras frecuencias en Onda Media o FM, que provienen en su mayoría de la absorción de ABC Punto Radio, para dar cobertura a retransmisiones deportivas locales. En el caso de Sevilla, COPE+ (emite a través de la frecuencia en OM 837 y FM 98.4) recoge toda la programación local en un magacín diario, de 12:20 a 13 horas, y diversos programas temáticos (toros, motor, cofradías...) en horario de tarde, además de las transmisiones de Semana Santa o los partidos de los clubes sevillanos. Esta opción de COPE+ es también una buena oportunidad para el departamento comercial, que permite a los pequeños anunciantes seguir teniendo presencia publicitaria en la Cadena a través de patrocinios de contenidos puramente locales.

\section{Presencia del ideario en la emisora}

Como expresa el propio ideario de la cadena, "El origen y la titularidad de la COPE marcan su identidad y sus propósitos. Como cadena radiofónica surgida de las Iglesias diocesanas y de dos órdenes religiosas en España, y asumida después por su Conferencia Episcopal, la Cadena se considera a sí misma como confesionalmente católica y se sitúa, de partida, en el marco de los fines generales de la Iglesia y, más en concreto, de su presencia evangelizadora en el ámbito de la opinión pública" [6].

Hoy en día, de acuerdo con su vocación y su ideario, COPE sostiene el valor de la familia, la promoción de la cultura de la vida y una sana laicidad que reconozca la aportación de la experiencia religiosa al bien común dentro de su programación generalista, con especial atención al magisterio del Papa Francisco y a la vida de las diócesis españolas. La línea editorial de la emisora se difunde diariamente en breves editoriales de opinión que se vierten junto a los informativos firmados como "Línea COPE" y realizados por José Luis Restán, director editorial de la emisora.

La Cadena COPE, fiel a su vocación e ideario, a través de sus diversos programas ha dado voz a las organizaciones de la sociedad civil, a las empresas y a las ONG's. COPE prioriza el valor indispensable de la familia, la promociona la cultura de la vida y una sana laicidad que reconozca la aportación de la experiencia religiosa al bien común. En su programación se refleja la presencia de la Iglesia en todos los campos (caridad, cultura, misiones), y se presta especial atención al magisterio del Papa Francisco y a la vida de las diócesis españolas.

\section{3.- ESTRUCTURA ACTUAL, ACCIONARIADO Y PARTICIPACIONES DEL GRUPO COPE EN OTROS MEDIOS}

\section{Accionariado y Consejo de Administración}

La Conferencia Episcopal posee un 51\% del accionariado de COPE. Las diócesis, por su parte, controlan otro $21,4 \%$ de las acciones. En tercer lugar se sitúa el expresidente del Real Madrid Vicente Boluda que aglutina el 6\% de la emisora y que, en 2015, compró 224.555 acciones por un importe de 4,5 millones de euros.

Fernando Giménez Barriocanal es Presidente y Consejero Delegado de Cadena COPE, además de Consejero de 13TV [7] y Presidente del Patronato de la Fundación COPE. Además, es vicesecretario de para asuntos económicos de la Conferencia Episcopal 
Española. Junto a él, el resto del consejo de administración del Grupo COPE lo componen [8]:

- Vicepresidente y Consejero Delegado: Rafael Pérez del Puerto Rodríguez

- Vicepresidente y Consejero: José María Mas Millet, en representación de TPL Cartera de Medios Audiovisuales, S.L.

- Antonio Mayor Villa, en representación de la Organización Nacional de Ciegos Españoles (ONCE).

- Vicente Boluda Fos, en representación de Boluda FosCorporation, S.L.

- Vicente Rebollo Mozos, en representación de Unión Mutua Asistencia de Seguros a Prima Fija (UMAS).

- Luis Colina Lordá, en representación de Diario de Navarra, S.A.

- Juan Díaz-Bernardo Navarro.

- Pedro Antonio Martín Marín.

- David Martín Casero.

- Ana María López García.

- José Gabriel Vera Beorlegui.

- Julia García Monge.

- Lydia Sanz Montes.

- Fernando A. Martínez García.

- José Ramón Gortázar Díaz (secretario).

Según las últimas cuentas aprobadas en 2017 por la Junta de Accionistas de la emisora [9], correspondientes al ejercicio 2016, la facturación del Grupo COPE alcanzó los 106 millones de euros, lo que representa un aumento del $14 \%$ respecto al ejercicio anterior. Esta cifra contrasta con el comportamiento general del sector de la radiodifusión en España, y ratifica, como suele destacar con especial énfasis la emisora cuando se trata de hacer públicas sus cuentas, "la sólida situación de la compañía y la ausencia de endeudamiento bancario" [10].

El Grupo presentó en 2016 un incremento de beneficios, que ascienden a 4 millones de euros, un crecimiento del $300 \%$ respecto al ejercicio anterior. Otro dato a destacar de las cuentas de 2016 es el EBITDA ${ }^{[11]}$ que alcanza los 7 millones de euros, y la existencia de un patrimonio neto de la emisora que representa el $56 \%$ del total del pasivo. La evolución económica de la empresa en el primer semestre de 2017 muestra una subida del $79 \%$ de las ventas en publicidad, superando los 4 millones de euros.

\section{Medios radiofónicos en los que participa el Grupo COPE}

El Grupo COPE, a través de la sociedad Radio Popular S.A., controla distintos medios radiofónicos además de la propia Cadena COPE [12]:

CADENA 100, que tiene su precedente en Popular FM, una cadena de emisoras musicales de Radio Popular que empezó a emitir el 2 de mayo de 1992, a partir de esa propia red de emisoras bajo la dirección de Rafael Revert, fundador de Los 40 Principales.

Megastar FM, que apuesta por los últimos éxitos internacionales y se encuentra claramente enfocada a un perfil juvenil. 
Rock FM, emisora especializada en música rock que inició sus emisiones en 2004 con el nombre de Rock \& Gol, con una programación temática centrada en los deportes y la música rock, hasta que en 2011 cambió su nombre y formato al actual.

Además, el Grupo COPE participa en [13]:

- Radio 13 de Cataluña S.A.

- Protemas S.A.

- Editorial Catalana S.A.

- Dial Doñana. S.L.

- Difusión Imagen y Voz S.A.

- FM Canarias S.L.

\section{La presencia en televisión: 13 TV}

Por otro lado, la participación del Grupo COPE en televisión se centra en el canal 13 TV de emisión nacional. Sus principales contenidos pasan por el cine clásico, las teleseries, los programas de producción propia y los de producción ajena religiosa. Debido a la entrada de la Conferencia Episcopal en el accionariado de 13TV, esta decidió integrar las emisiones de su red de televisiones regionales Popular TV en 2011, para abaratar costes y no mantener por más tiempo programación duplicada en ambas cadenas. Actualmente está operado por 13TV S.A. y pertenece al Grupo Radio Popular.

Su accionariado está controlado en un $80 \%$ por la Conferencia Episcopal, un $12 \%$ lo posee Sandwich (José María MasMillet, persona de confianza del Cardenal Osoro y a la sazón presidente de 13 TV), el 11\% está en manos de Betafilm, un 7\% es de Spinnaker, el 5\% lo posee Videomercury, el $4 \%$ Seguros UMAS, que también participa en COPE al igual que el Arzobispado de Madrid que tiene un 3\% del accionariado. El 1\% es de UJUE Producciones, cuyo propietario Alejandro Samanés, fundador de la cadena.

Aunque su devenir merecería un profundo análisis en otro trabajo exclusivamente dedicado al canal, debemos hacer una serie de consideraciones al respecto. La situación actual de 13 TV dista mucho de la que vive la Cadena COPE [14]. Las importantes pérdidas económicas anuales de la televisión y el escaso nivel de audiencia de los programas sitúan anualmente a 13 TV en una comprometida situación de cara a las juntas de accionistas del Grupo COPE. No son pocas las ocasiones en las que los portales de información se hacen eco de la situación financiera de la cadena episcopal, teniendo en cuenta que los últimos ejercicios se han cerrado con déficit —aminorado, eso sí, en los últimos años - y las continuas inyecciones de dinero que se han hecho para salvar la cadena.

\section{REFERENCIAS}

GARRIDO BUSTAMANTE, José Luis. (1993). Sevilla tras un micrófono: Crónica y peripecias de la radio en la ciudad. Sevilla: Editorial Castillejo.

GÓMEZ Y MÉNDEZ, José Manuel, et al. (2006). Radio Vida, en el recuerdo de Sevilla. Sevilla: Publicaciones de la Universidad de Sevilla.

Web del Grupo COPE: www.cope.es. 
[1] "Radio Sevilla la nueva", la llamaban los sevillanos en lenguaje popular, según recoge el periodista José Luis Garrido Bustamante en GARRIDO BUSTAMANTE, José Luis. (1993). Sevilla tras un micrófono: Crónica y peripecias de la radio en la ciudad. Sevilla: Editorial Castillejo.

[2] Solo interrumpida entre los años 2007-2009, aunque el espíritu del programa no se desvaneció del todo con el galardón "Saeta de Oro", una de las más preciadas distinciones del mundo de las hermandades, y al crear la emisora una publicación cuaresmal bajo la cabecera de Saeta que actualmente se sigue editando con la dirección de quien suscribe. Además, las propias transmisiones cofradieras se emiten bajo la cabecera de Saeta en Semana Santa.

[3] Ocupaba la cartera del citado ministerio Manuel Fraga Iribarne; era subsecretario del departamento Pío Cabanillas.

[4] La operación fue anulada por el Tribunal Supremo. PRISA convirtió las emisoras de Antena 3 en una cadena de música clásica, Sinfo Radio.

[5] Luis del Olmo abandonó COPE en 1991 para incorporarse a Onda Cero. Fue uno de los principales motivos por los que la emisora empezó a perder audiencia.

[6] Puede consultarse el ideario completo en la web http://www.cope.es/pagina/ideario.

[7] Dentro de la emisora, cuanto a 13TV y según datos ofrecidos por la propia cadena, su Presidente es José María Mas; Alejandro Samanes es el Consejero Director General de la televisión; José Pablo López es el director de la misma, mientras que José Gabriel Vera es Consejero (al igual que el Consejero Delegado de Cadena Cope, Giménez

Barriocanal)además de Director del Secretariado de la Comisión Episcopal de Medios de Comunicación Social; Juan Pedro Ortuño también realiza funciones de Consejero, aparte de delegado de medios del Arzobispado de Madrid

[8] Componentes del Consejo a la celebración de la última junta de accionistas de junio de 2017.

[9] Información publicada por la propia emisora a través de su web:

http://www.cope.es/noticias/actualidad/grupo-cope-aumenta-facturacion-2016_152041

[10] Íb.

[11] EI EBITDA (acrónimo del inglés "EarningsBeforelnterest, Tax, Depreciation and Amortization") es un indicador que mide el margen bruto de explotación de la empresa antes de deducir los intereses, impuestos y amortizaciones.

[12] Una nota para destacar, al igual que se ha hecho anteriormente con los datos de la Cadena COPE, los datos del EGM para las emisoras musicales del grupo: CADENA 100, Rock FM y MegaStar FM también siguen una línea alcista. CADENA 100 ha registrado el mejor dato de toda su historia con más de 2.100 .000 oyentes diarios. 
[13] La emisora Herrilrratia estuvo asociada a COPE hasta el 25 de marzo de 2010, cuando la cadena fue vendida al Grupo Noticias Onda Vasca. El 15 de septiembre de ese año, también Gestiona Radio dejó de estar vinculada al Grupo COPE, al vender su participación del $70 \%$ por 140.000 euros. El motivo de la venta interés de COPE de centrarse exclusivamente en su producto en un entorno en crisis.

[14] La información publicada por el portal El Confidencial en 2016:

https://www.elconfidencial.com/comunicacion/2016-10-22/la-iglesia-no-gana-para-susmedios-y-entierra-89-millones-en-cope-y-13tv-desde-2010_1278553/

\section{BREVE SEMBLANZA DEL AUTOR:}

Pedro Domínguez Fernández es licenciado en Periodismo por la Universidad de Sevilla. Ha sido Redactor de informativos y presentador en Giralda TV (televisión municipal de Sevilla). Vinculado profesionalmente a la COPE desde 2004, actualmente es director de las transmisiones de la Semana Santa de esta emisora de radio. Es profesor del máster de periodismo de la Cámara de Comercio de Sevilla.

- Recibido: 08/10/2017

- Aceptado: 02/02/2018

Ámbitos. Revista Internacional de Comunicación, n.40, edición de primavera, 2018. 